\title{
A systematic review and meta-analysis of the effect of different doses of rocuronium for general anesthesia on thyroid surgery
}

\author{
Bihua Zhong', Guofang Gao ${ }^{2}$, Dan Sun ${ }^{2}$, Lixia Zhang ${ }^{3}$ \\ ${ }^{1}$ Department of Pharmacy, Xiaoshan District Women and Children's Health Service Center, Hangzhou, China; ${ }^{2}$ Department of Pharmacy, The First \\ People's Hospital of Xiaoshan District, Hangzhou, China; ${ }^{3}$ Outpatient Department, The First Affiliated Hospital, Zhejiang University, Hangzhou, \\ China \\ Contributions: (I) Conception and design: B Zhong, G Gao; (II) Administrative support: D Sun; (III) Provision of study materials or patients: B \\ Zhong, D Sun, L Zhang; (IV) Collection and assembly of data: All authors; (V) Data analysis and interpretation: G Gao, L Zhang; (VI) Manuscript \\ writing: All authors; (VII) Final approval of manuscript: All authors. \\ Correspondence to: Lixia Zhang. Outpatient Department, The First Affiliated Hospital, Zhejiang University, Hangzhou, China. Email: abbyzjj123@163.com.
}

Background: The most common complication of thyroid anesthesia is recurrent laryngeal nerve injury, and anesthesia drugs affect the intraoperative recurrent laryngeal nerve monitoring indicators, especially nerve blockers, which can cause muscle relaxation and affect vocal cord contraction. The purpose of this study was to investigate the optimal dose of rocuronium during general anesthesia (GA) during thyroid surgery by meta-analysis.

Methods: Chinese and English databases were searched by the combination of "thyroid", "general anesthesia", and "rocuronium bromide". Publications which took double dose rocuronium GA surgery as the double group and normal dose rocuronium GA surgery as the normal group were included, and Review Manager 5.3 (Rev Man 5.3) was employed for meta-analysis.

Results: A total of fifteen publications were included in the meta-analysis and an overall heterogeneity test was conducted to obtain the results. Comparison of the operation time between the double group and the normal group showed the mean difference (MD): $20.93,95 \%$ confidence interval (CI): $(11.48,30.39) \mathrm{min}$, $\mathrm{Z}=4.34, \mathrm{I}^{2}=94 \%$, and $\mathrm{P}<0.0001$, while that of bleeding volume between the double group and the normal group showed the MD: $-24.34,95 \%$ CI: $(-28.11,-20.58) \mathrm{mL}, \mathrm{Z}=12.67, \mathrm{I}^{2}=91 \%$, and $\mathrm{P}<0.0001$. Comparison of drainage volume between the double group and the normal group showed the MD: 24.40, 95\% CI: (19.84, 28.96) $\mathrm{mL}, \mathrm{Z}=10.49, \mathrm{I}^{2}=68 \%$, and $\mathrm{P}<0.0001$, and between hospitalization days between the double group and the normal group showed the MD: $-13.50,95 \%$ CI: $(-18.02,-8.97)$ days, $Z=5.85, I^{2}=99 \%$, and $P<0.0001$. Finally, comparison of satisfaction between the double group and the normal group showed risk ratio (RR): $2.16 \%, 95 \%$ CI: $(1.88 \%, 2.48 \%), Z=10.91, \mathrm{I}^{2}=0$, and $\mathrm{P}<0.0001$. Rev Man 5.3 was employed to obtain the funnel chart of each observation indicator, and the circles of some publications were concentrated on the midline and symmetrical, indicating the research accuracy was high and there was no bias in the publications. Discussion: This meta-analysis confirmed that a double dose of rocuronium can meet the needs of anesthesia induction and GA during thyroid surgery.

Keywords: Thyroid; general anesthesia (GA); rocuronium

Submitted Apr 10, 2021. Accepted for publication Sep 24, 2021.

doi: $10.21037 / \mathrm{gs}-21-618$

View this article at: https://dx.doi.org/10.21037/gs-21-618 


\section{Introduction}

Thyroid disease is common, and its annual incidence is rising (1). Thyroid diseases that require surgical intervention include hyperthyroidism, thyroid cancer, thyroid tumor, and nodular goiter. Damage to the recurrent laryngeal nerve is a common complication of thyroid surgery (2), and is caused by ligation, traction, or cutting of nerve bundles (3). Unilateral damage to the nerve may cause hoarseness, while bilateral damage may cause breathing difficulties (4). Studies have shown the incidence of recurrent laryngeal nerve injury during surgery to be about $1.2-10.1 \%$, and if surgery was performed again after recurrence, the incidence was as high as $18.3-35.6 \%$ (5). The monitoring of the function of recurrent laryngeal nerve during thyroid surgery plays a key role in preventing postoperative complications, and narcotic drugs have a great influence on the monitoring of the function of recurrent laryngeal nerve. Different concentrations of rocuronium have different effects on electromyography (EMG) recovery, so it is urgent to find the most suitable dose to meet the needs of anesthesia and monitoring of recurrent laryngeal nerve.

There are currently two main anesthesia methods for thyroid surgery, one of which is cervical plexus anesthesia. During this type of operation, the patient is awake and can communicate during the procedure. Moreover, surgical staff can observe the patient's pronunciation and damage to the nerve in real time (6). Despite this, the procedure is rarely used because of the stress being conscious causes to the patient (7). Another method of anesthesia is general anesthesia (GA), which induces intubation through the injection of small doses of non-depolarizing muscle relaxants, including rocuronium bromide (8).

Since Lu et al. [2011] (9) proposed the use of rocuronium for GA during thyroid surgery in 2015, the dosage has been controversial. Hurford et al. [2020] (10) conducted a metaanalysis and found that in 25 clinical randomized controlled double groups, different doses of rocuronium had different effects. Continuous advances have resulted in a wealth of publications in the field, and it is necessary to evaluate current literature to provide a new diagnostic basis for clinical thyroid surgery.

The innovation of this study was that systematic evaluation and meta-analysis were used to compare the EMG recovery of patients undergoing twice-dose rocuronium GA surgery and normal rocuronium GA surgery, so as to explore the best dose of rocuronium in thyroid surgery and avoid adverse reactions caused by intravenous anesthesia. We present the following article in accordance with the PRISMA reporting checklist (available at https://dx.doi.org/10.21037/gs-21-618).

\section{Methods}

\section{Literature searching}

We searched the PubMed, MEDLINE, Embase, Chinese Biomedical Literature Database, China National Knowledge Network database, WanFang database, VIP database, and Google Academic databases. The retrieval time was from establishment to November 25th, 2020, and composite logic retrieval and Boolean logic retrieval were used to select relevant literature. Chinese and English databases were searched by the combination of "thyroid", "general anesthesia", and "rocuronium bromide". All kinds of search words were freely combined, and after searching for certain documents for many times, the search engine was used to trace the certain documents. The quality of literature was evaluated using Review Manager 5.3 (Rev Man 5.3) provided by the Cochrane system.

\section{Inclusion and exclusion criteria of publications}

The inclusion criteria for publications were as follows: (I) randomized controlled trials to explore the effect of rocuronium on thyroid GA surgery; (II) subjects were 18 years or older; (III) the experiment group included patients with GA by intravenous rocuronium; (IV) thyroid diseases were diagnosed by World Health Organization.

The exclusion criteria were any of the following: (I) research objects overlapped each other; (II) repeated published articles; (III) animal experiments or thyroid tumor cell experiments; (IV) research that can't contact the original author or obtain complete data; (V) research literature on non-anesthesia surgery.

\section{Measurement indicators and intervention measures for thyroid GA surgery}

The measurement indicators used were operation time $(\mathrm{min})$, intraoperative blood loss $(\mathrm{mL})$, postoperative drainage $(\mathrm{mL})$, hospital stay $(\mathrm{d})$, and patient satisfaction.

The intervention measures in the double group were a double dose of rocuronium anesthesia for thyroid surgery, and in the normal group this was a normal dose of rocuronium. 


\section{Data extraction}

The two researchers independently read the title, abstract, and full text of the research, excluded the unqualified documents, and read the full text of the documents meeting the inclusion criteria to obtain relevant information. If duplicate studies were found and published, the latest or most comprehensive ones would be included. If the research data was incomplete, researcher can further contact the author of the research to obtain relevant data, and eliminate the research that had not obtained relevant data in the end. Finally, the randomized controlled trials were selected, and the data were extracted and then cross-checked. If there was any disagreement among researchers, the consensus conclusion was obtained through discussion.

\section{Bias risk assessment}

The Cochrane Handbook 5.1 collaboration network was used as a tool for "risk of bias assessment" for randomized controlled trials. The evaluation criteria included generation of random sequence, blind method, hiding of allocation scheme, integrity of data results, and research results. The above five aspects were judged, respectively, as "high risk bias", "low risk bias", and "unclear".

\section{Literature quality assessment}

In this study, the GRADE standard in the Cochrane Collaboration was used for quality classification.

\section{Statistical analysis}

Stata SE 12.0 was employed for statistical analysis. Mean difference (MD), risk ratio (RR), and 95\% confidence interval (CI) were used to evaluate the statistics of rocuronium intravenous GA and Rev Man 5.3's risk of bias assessment chart was utilized to assess the risk bias. Each effect was expressed using a $95 \% \mathrm{CI}$, and when the heterogeneity test showed that $\mathrm{P}>0.01$ and $\mathrm{I}^{2}<50 \%$, the fixed effects model was used for meta-analysis. When the heterogeneity test showed that $\mathrm{P}<0.01$ and $\mathrm{I}^{2}>50 \%$, the random effects model was used for meta-analysis.

\section{Sensitivity analysis}

The influence on the total combined effect was studied, and the research with great influence was excluded. The results of random effect model and fixed effect model were compared, and the reliability of the combined results were analyzed and reflected according to the consistency of the results. Funnel chart was used to judge whether there was publication bias.

\section{Results}

\section{Search results and basic information of included literature}

In total, 427 literatures were obtained by searching the database, 438 literatures were obtained by searching the register, 129 literatures published repeatedly were excluded, 75 literatures were rejected as unqualified, 38 literatures were excluded for other reasons, and the remaining 623 literatures were obtained. Three hundred and seventeen duplicated subjects were excluded, leaving 306 . The remaining 50 articles were excluded from 256 reviews. Thirty-nine reports were eliminated. Thirty-six papers were retrieved from websites, 27 from scientific research institutions, and 18 from citation retrieval. After repeated screening, 46 articles were included, 35 reviews were excluded and 11 articles remained. Seven reports were excluded. After multiple combination screening, 15 literatures were finally included (Figure 1).

The 15 publications which met the inclusion criteria included 1,290 patient cases and all involved a blank normal group. All publications were small-sample studies, ranging from 28 to 112, and all research subjects were over 18 years old. The age, operation time, blood loss, drainage volume, and length of hospital stay in the double group and normal group were described in 11 publications. Basic information on the research objects is shown in Table 1 .

\section{Results of literature risk bias evaluation}

Figures 2,3 show the results of multiple risk bias evaluations of the publications drawn by Rev Man 5.3. Among the 15 randomized controlled experiments, three (11-13) described the correct random allocation method, two $(14,15)$ described the correct random allocation method and described the concealment of the allocation plan in detail, one (16) was evaluated by a blind method, and the other (17-25) was unblinded. However, as the measurement indicators in the latter work were laboratory indicators determined by computer, all articles were considered to be blinded correctly.

\section{Comparison of operation time}

A total of six publications analyzed the operation time of 
Identification of studies via databases and registers
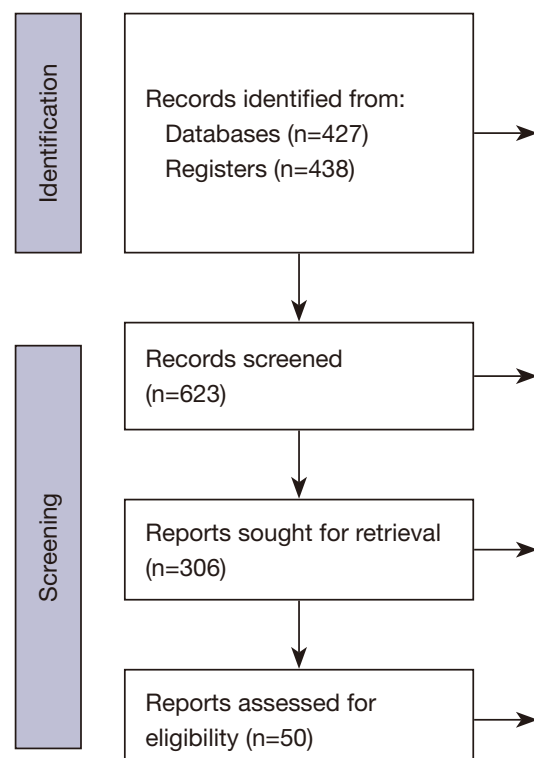
$(n=623)$
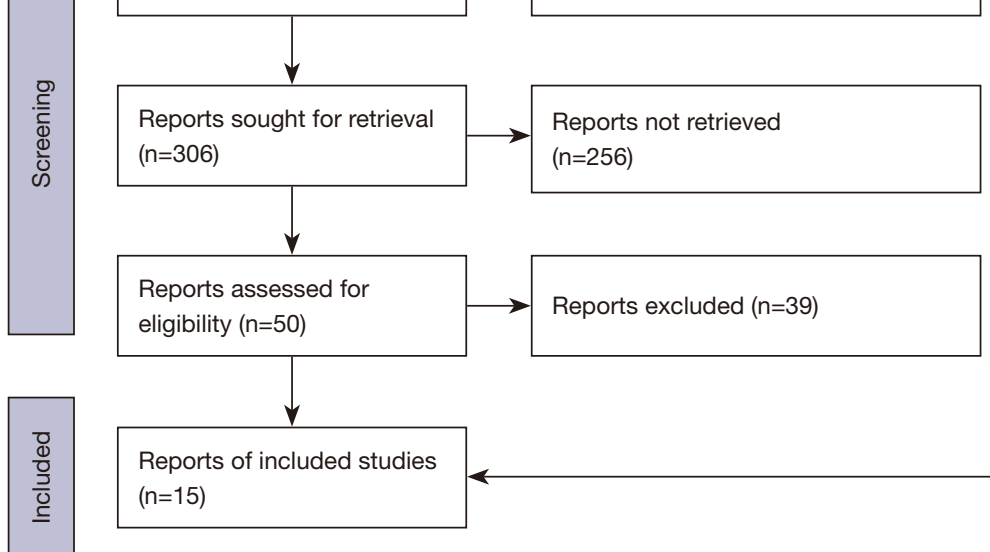
$(n=256)$

Records removed before screening: Duplicate records removed $(n=129)$ Records marked as ineligible by automation tools $(n=75)$ Records removed for other reasons $(n=38)$

Records excluded $(n=317)$

Reports excluded $(n=39)$

\section{Identification of studies via other methods}
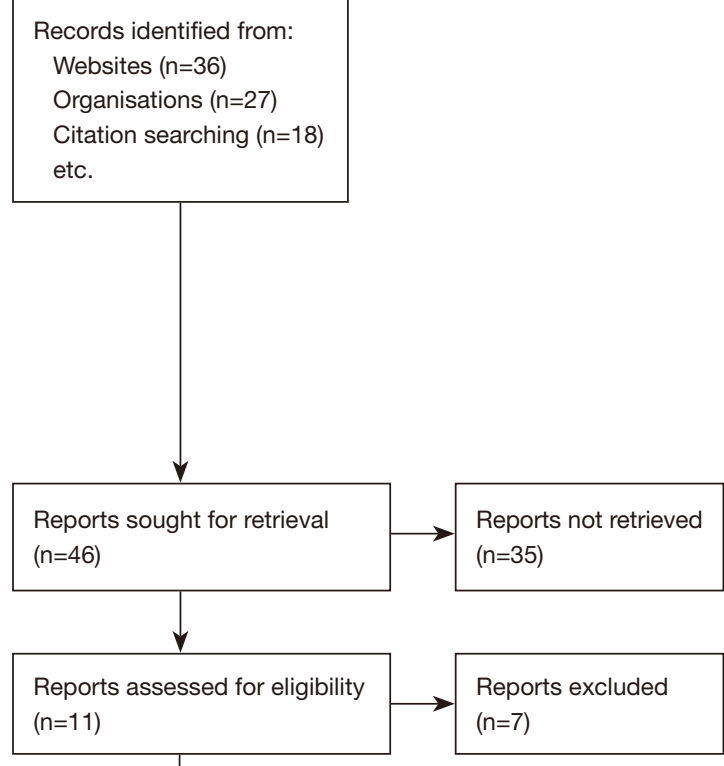

Figure 1 Search flow chart.

Table 1 General information of the included literature

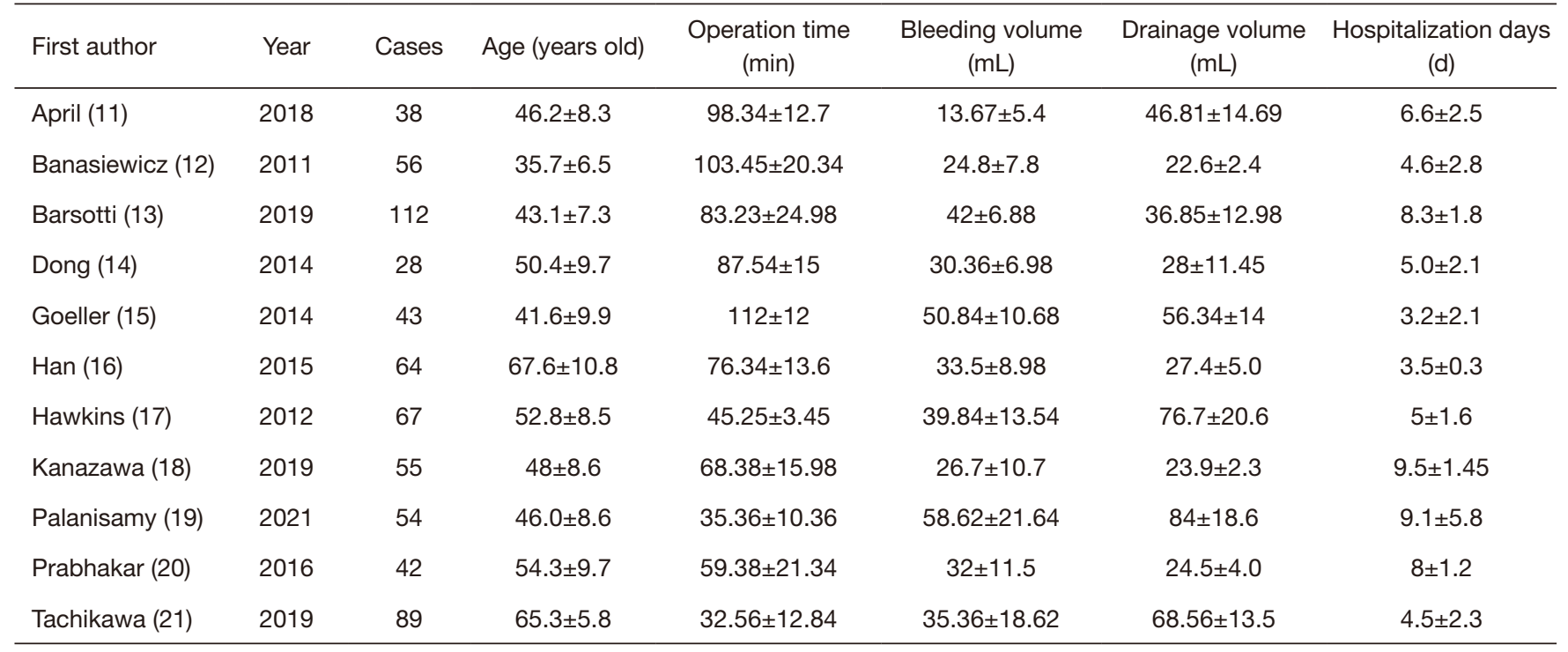




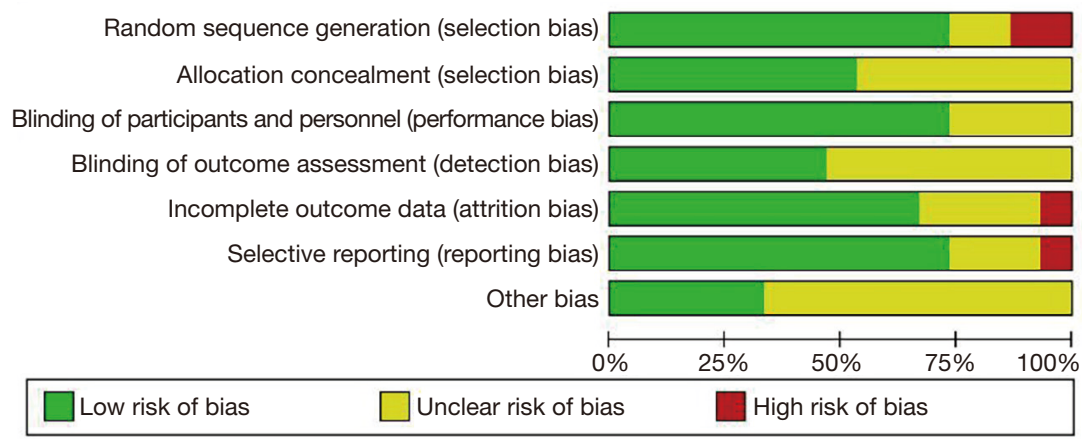

Figure 2 Literature risk bias evaluation results.

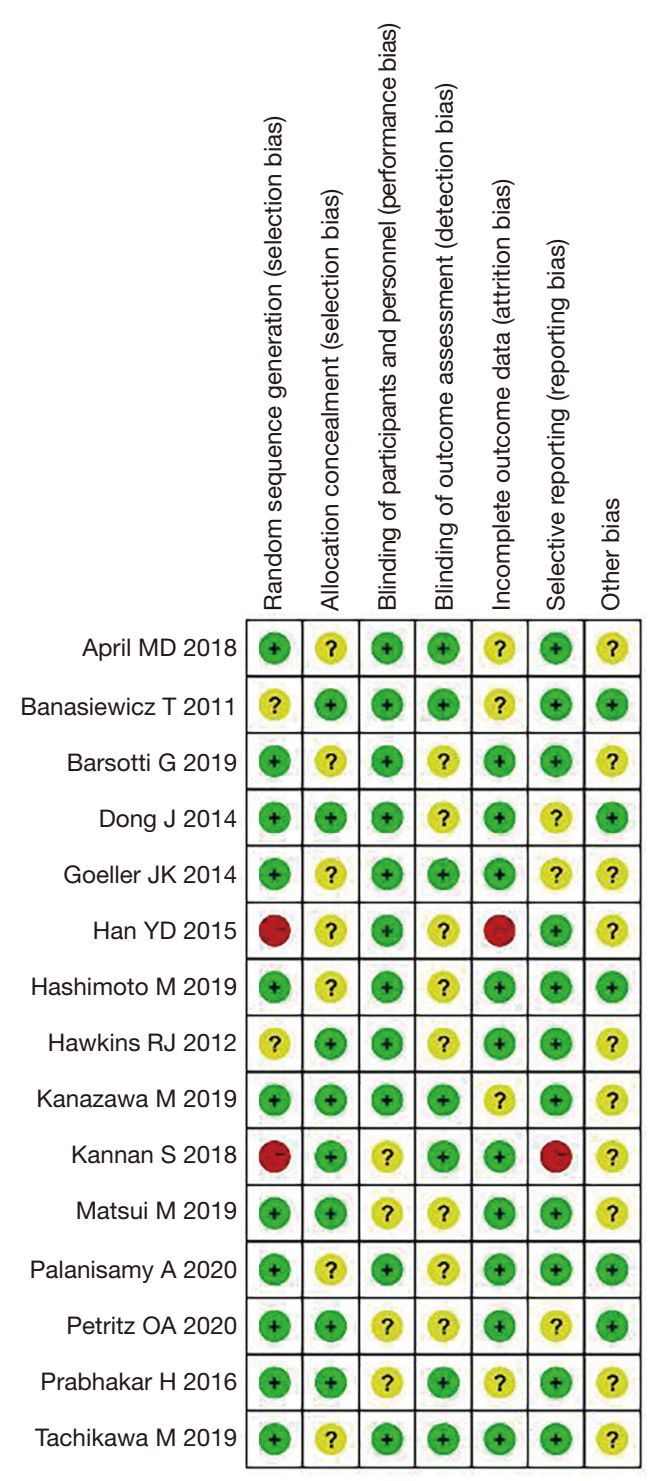

Figure 3 Bias evaluation results of included literature corresponding to multiple risks. rocuronium GA in randomized controlled trials. There were 785 cases in total, 393 cases in the double group, and 392 cases in the normal group. The overall heterogeneity showed the MD: 20.93, 95\% CI: $(11.48,30.39) \mathrm{min}$, $\mathrm{Z}=4.34, \mathrm{I}^{2}=94 \%$, and $\mathrm{P}<0.0001$, and the horizontal line of the $95 \% \mathrm{CI}$ of all publications fell on the right side of the invalid vertical line. The random effects model was used for meta-analysis, and the difference between the double group and the normal group was substantial (Figure 4).

The inverted funnel chart of the operation time data obtained using Rev Man 5.3 showed that the data was relatively concentrated. Moreover, the circles of some publications were basically symmetrical with the midline, suggesting the research accuracy was high and there was no bias in the publications (Figure 5).

\section{Comparison of surgical bleeding}

A total of six publication analyzed the bleeding volume of rocuronium GA in randomized controlled trials. There were 785 cases, with 393 in the double group and 392 in the normal group. The overall heterogeneity test was performed and showed the MD: $-24.34,95 \% \mathrm{CI}$ : $(-28.11,-20.58) \mathrm{mL}, \mathrm{Z}=12.67, \mathrm{I}^{2}=91 \%$, and $\mathrm{P}<0.0001$, and the heterogeneity between the double groups was relatively large. The horizontal lines of the $95 \%$ CI of the publications all fell to the right of the invalid vertical line. The random effects model was used for meta-analysis, and the difference between the double group and the normal group was substantial (Figure 6). It indicated that the amount of bleeding in the double group was significantly lower than that in the normal group.

The inverted funnel chart of the surgical bleeding data showed that the data was relatively concentrated. Moreover, the circles of some publications were basically symmetrical 


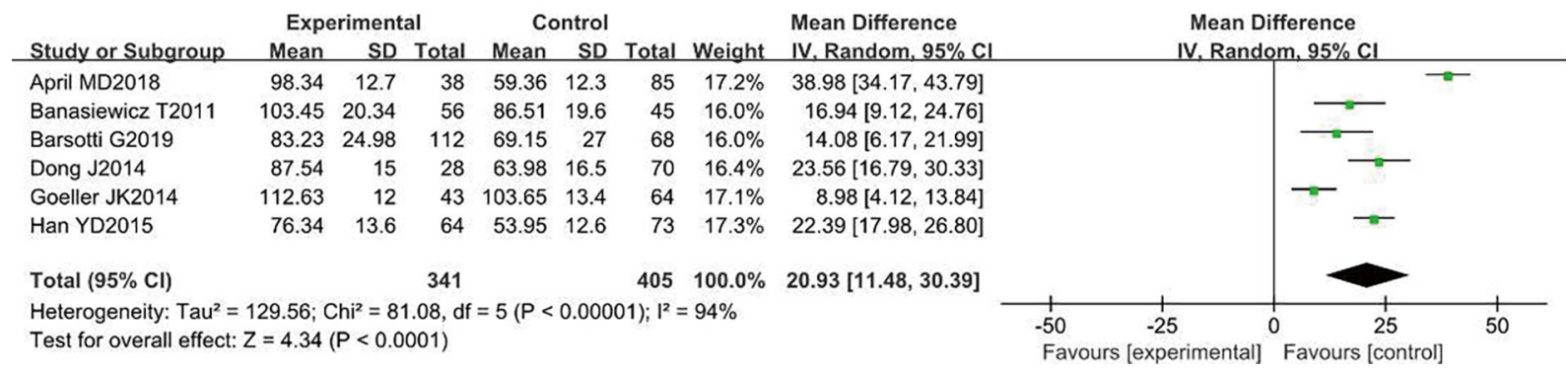

Figure 4 Comparison of operation time between double group and normal group. CI, confidence interval.

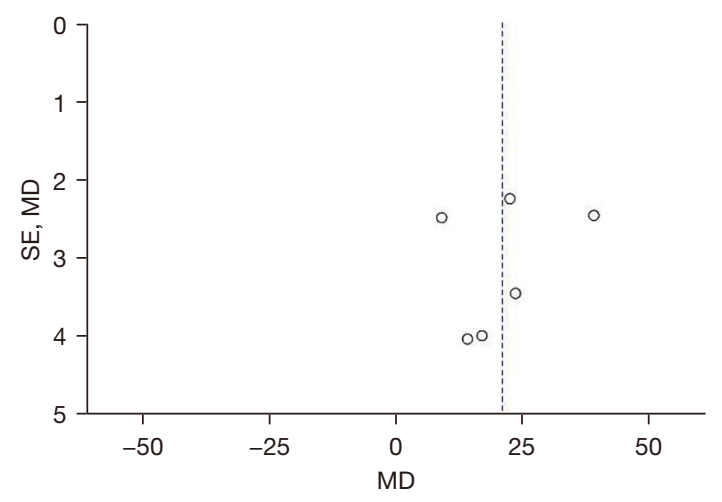

Figure 5 Funnel chart of comparison of operation time between the double group and the normal group. SE, standard error; MD, mean difference.

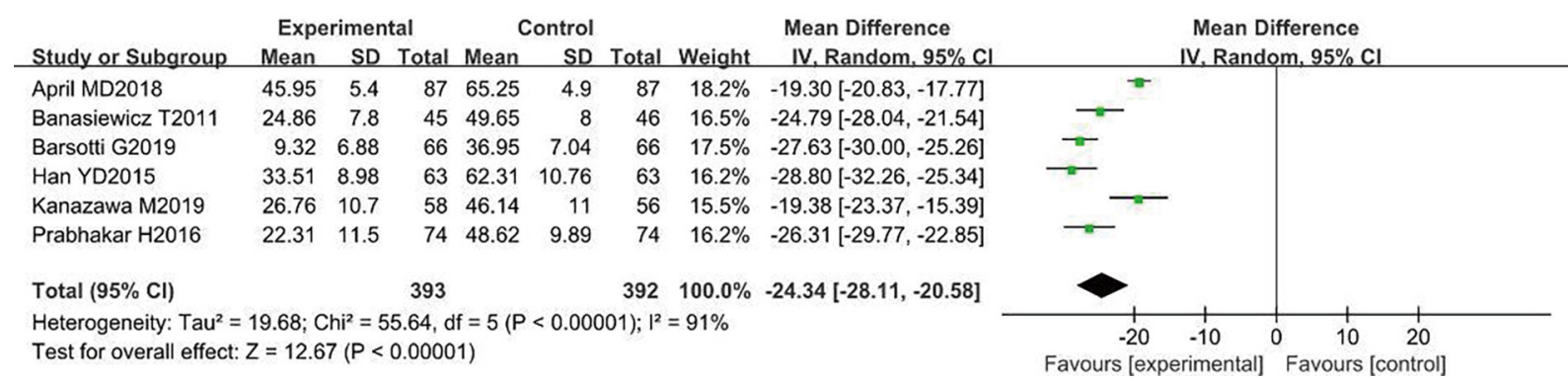

Figure 6 Comparison of blood loss between double group and normal group. CI, confidence interval.

with the midline, suggesting that the research accuracy was high and there was no bias in the publications (Figure 7).

\section{Comparison of drainage volume}

The drainage volume of rocuronium GA in randomized controlled trials was analyzed in five publications. There were 514 cases in total, with 256 in the double group and 258 in the normal group. The overall heterogeneity test showed the MD: $24.40,95 \%$ CI: $(19.84,28.96) \mathrm{mL}$, $\mathrm{Z}=10.49, \mathrm{I}^{2}=68 \%$, and $\mathrm{P}<0.0001$, and the heterogeneity between the double groups was relatively large. The horizontal lines of the $95 \%$ CI of the publications all fell to the right of the invalid vertical line. The random effects model was used for meta-analysis, and the difference between the double group and the normal group was substantial (Figure 8).

The inverted funnel chart of the drainage volume data 


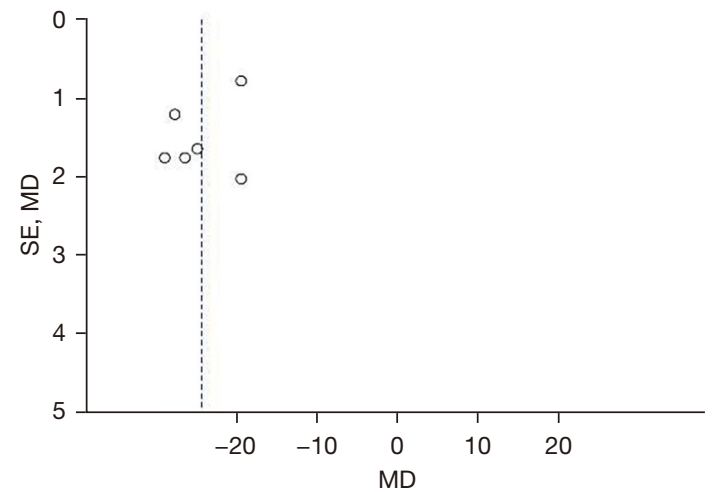

Figure 7 Funnel chart of comparison of blood loss between double group and normal group. SE, standard error; MD, mean difference.

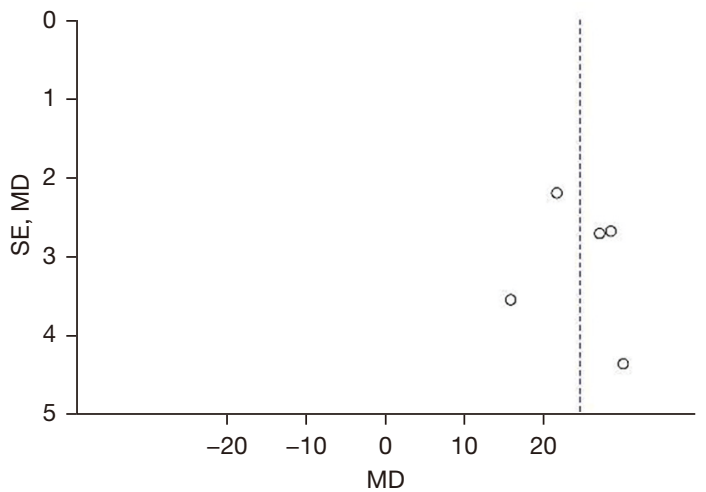

Figure 9 Funnel chart of comparison of drainage volume between double group and normal group. SE, standard error; MD, mean difference.

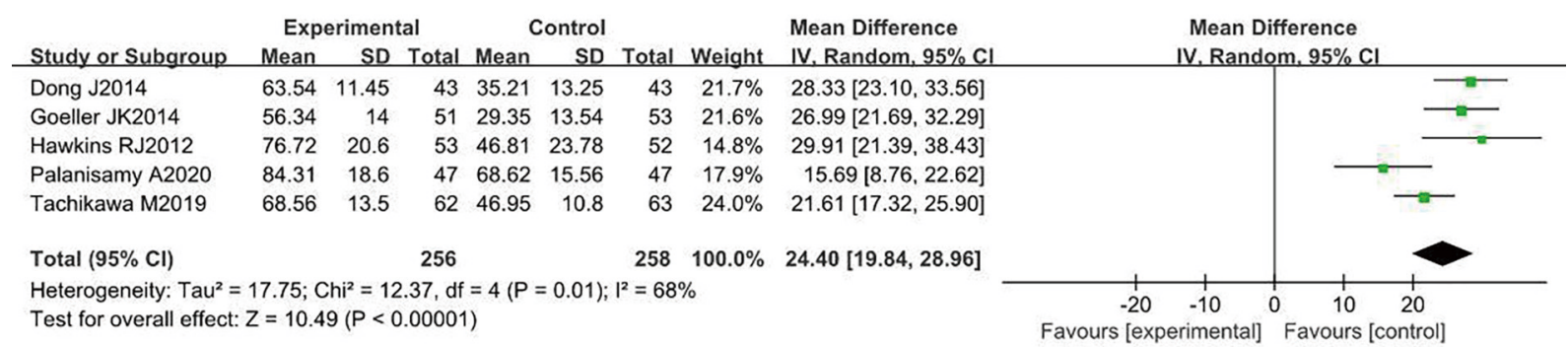

Figure 8 Comparison of drainage volume between double group and normal group. CI, confidence interval.

showed that the data was relatively concentrated. Moreover, the circles of some publications were basically symmetrical with the midline, suggesting that the research accuracy was high and there was no bias in the publications (Figure 9).

\section{Comparison of hospitalization days}

Hospitalization days for GA with rocuronium were analyzed in a randomized controlled trial which was discussed in four publications. There were 372 cases in total, with 186 cases in each of the double group and the normal group. The overall heterogeneity test showed the MD: $-13.50,95 \%$ CI: (-18.02, -8.97) days, $\mathrm{Z}=5.85, \mathrm{I}^{2}=99 \%$, and $\mathrm{P}<0.0001$, and heterogeneity between the double groups was relatively large. The horizontal lines of the $95 \%$ CI of the publications all fell to the left of the invalid vertical line. The random effects model was used for meta-analysis, and the difference between the double group and the normal group was substantial (Figure 10). The recovery rate of patients in the double group was faster than that in the normal group.

The inverted funnel chart of the hospitalization days data obtained using Rev Man 5.3 showed the data was relatively concentrated. Moreover, the circles of some publications were basically symmetrical with the midline, suggesting the research accuracy was high and there was no bias in the publications (Figure 11).

\section{Comparison of satisfaction}

Four publications analyzed the satisfaction of GA with rocuronium in randomized controlled trials. There were 465 cases in total, with 230 in the double group and 235 in the normal group. The heterogeneity test showed, RR: $2.16 \%, 95 \%$ CI: $(1.88 \%, 2.48 \%), \mathrm{Z}=10.91, \mathrm{I}^{2}=0$, and $\mathrm{P}<0.0001$, and the horizontal lines of the $95 \% \mathrm{CI}$ of the publications all fell to the right of the invalid vertical line. The random effects model showed the difference between the double group and the normal group was substantial (Figure 12). 


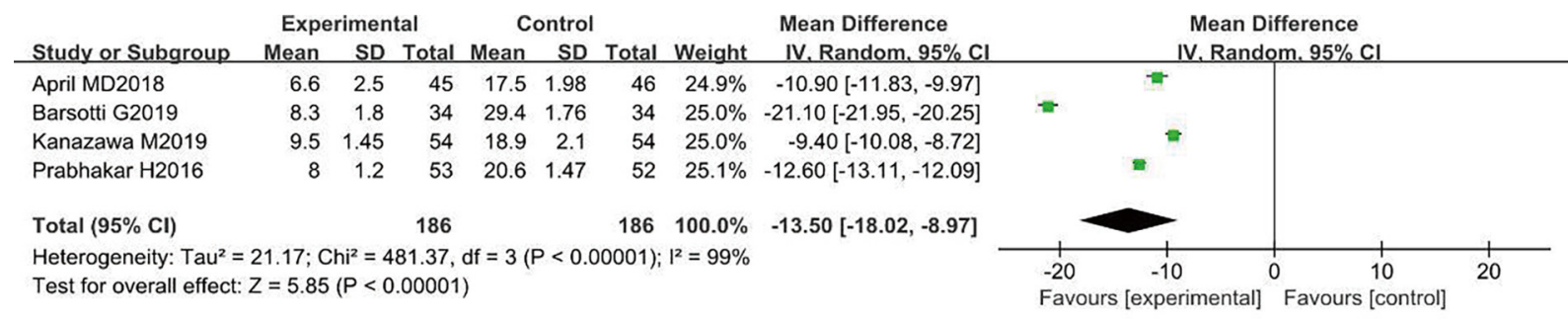

Figure 10 Comparison of hospitalization days between the double group and the normal group. CI, confidence interval.

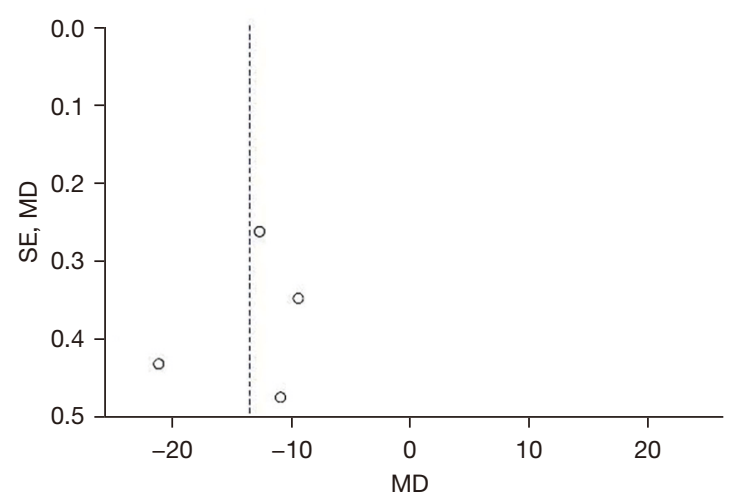

Figure 11 Funnel chart of comparison of hospitalization days between the double group and the normal group. SE, standard error; MD, mean difference.

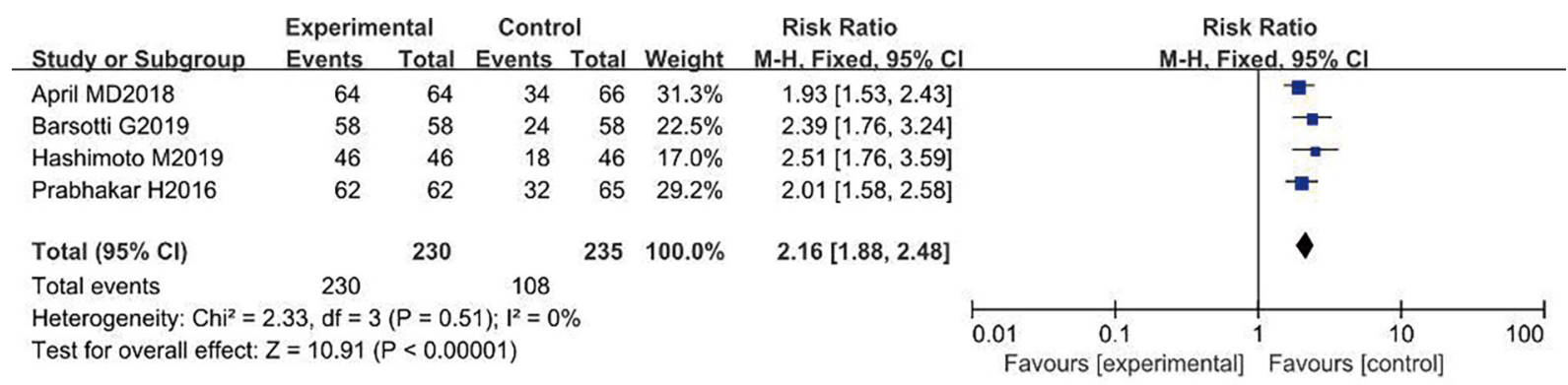

Figure 12 Satisfaction comparison between double group and normal group. CI, confidence interval.

The inverted funnel chart of the satisfaction data showed the data was relatively concentrated, and the circles of some publications were basically symmetrical with the midline, suggesting the research accuracy was high and there was no bias in the publications (Figure 13).

\section{Discussion}

The incidence of thyroid diseases is increasing, and surgery is the most common treatment (22-24). In case of recurrent laryngeal nerve injury, suture should be done immediately. In case of bilateral recurrent laryngeal nerve injury and besides tracheotomy, surgical exploration should be performed as soon as possible, and cartilage resection and vocal cord fixation should be performed if necessary to enlarge glottis. In order to avoid injury of recurrent laryngeal nerve during thyroid surgery, people should accumulate experience, familiarize ourselves with thyroid anatomy, improve surgical skills and the anatomical knowledge related to recurrent laryngeal nerve. During 


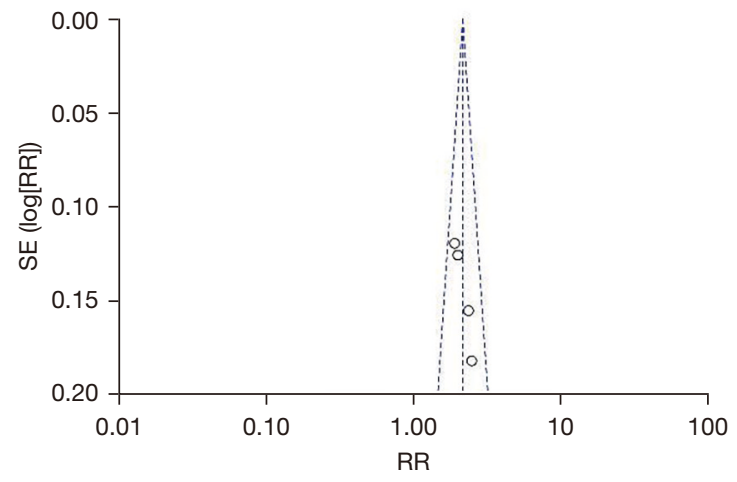

Figure 13 Funnel chart of the satisfaction comparison between the double group and the normal group. SE, standard error; RR, risk ratio.

thyroidectomy, dissecting recurrent laryngeal nerve should be avoided and posterior capsule should be kept as much as possible. When treating the inferior thyroid artery, it should be closely attached to the thyroid capsule, so as to avoid ligation of the inferior thyroid pole surface and blood vessels. When suturing the residual gland tissue, it should avoid penetrating the posterior capsule and accidentally injuring the recurrent laryngeal nerve (25-27). In this study, a meta-analysis was performed to compare double-dose and normal dose rocuronium in GA thyroid surgery. Since this was a retrospective analysis, the quality of the included literature was ideal, and according to the characteristics of the surgery, a double-blind method could be used.

The operation time, bleeding volume, drainage volume, hospitalization days, and satisfaction of patients undergoing a normal $(0.3 \mathrm{mg} / \mathrm{kg})$ or double dose of rocuronium $(0.6 \mathrm{mg} / \mathrm{kg})$ for GA were compared. After the random effects models was adopted, the results showed that the difference between the double group and the normal group was substantial and considerable. The overall heterogeneity test for the comparison of operation time showed the MD: 20.93, 95\% CI: $(11.48,30.39) \mathrm{min}, Z=4.34, \mathrm{I}^{2}=94 \%$, and $\mathrm{P}<0.0001$, and for the comparison of bleeding volume the MD: $-24.34,95 \%$ CI: $(-28.11,-20.58) \mathrm{mL}, Z=12.67$, $\mathrm{I}^{2}=91 \%$, and $\mathrm{P}<0.0001$. Comparison of drainage volume between the double group and the normal group showed the MD: $24.40,95 \%$ CI: $(19.84,28.96) \mathrm{mL}, Z=10.49$, $\mathrm{I}^{2}=68 \%$, and $\mathrm{P}<0.0001$, and for hospitalization days the MD: $-13.50,95 \%$ CI: $(-18.02,-8.97)$ days, $Z=5.85, I^{2}=99 \%$, and $\mathrm{P}<0.0001$. For the comparison of satisfaction between the double group and the normal group, RR: $2.16 \%, 95 \%$ CI: (1.88\%, 2.48\%), $\mathrm{Z}=10.91, \mathrm{I}^{2}=0$, and $\mathrm{P}<0.0001$. Rev Man 5.3 was employed to obtain the funnel chart of each observation indicator, and the circles of some publications were concentrated on the midline and basically symmetrical with the midline, which indicated the research accuracy was high and there was no bias in the publications. Aragón-Benedí et al. [2021] (28) conducted a meta-analysis which showed that compared with a normal group, twice the amount of rocuronium could meet the needs of anesthesia surgery.

We found that most of the included publications were in English. By comparing each indicator between the double group and the normal group, we found that the double group had obvious advantages in all aspects. While there was a great difference in the amount of bleeding between the groups, this result must be viewed with caution as the calculation methods were not clearly described in the publications and there may have been differences in the methods used. The patients listed in this study were mainly middle-aged and elderly patients who underwent thyroid surgery under GA, and there are some differences in studies of adolescents and patients without GA. In addition, the sample size included in the meta-analysis was small, so the scope of adoption of this study was limited to some extent.

\section{Conclusions}

In this study, a compound logic search method was used to retrieve 11 articles that took a double dose of rocuronium as an anesthetic agent as a double group and a normal dose of rocuronium as a normal group. A meta-analysis was then conducted to explore the effect of different rocuronium doses on thyroid surgery under GA. The results confirmed that a double dose of rocuronium can meet the needs of anesthesia induction and GA surgery. The limitation of this study lies in the high heterogeneity of the research objects and the small sample size of the included literature. Therefore, it is necessary to expand the sample size to conduct clinical randomized controlled experiments for further verification. To sum up, this study confirms that a double rocuronium dose has a significant advantage in thyroid surgery under GA.

\section{Acknowledgments}

Funding: None.

\section{Footnote}

Reporting Checklist: The authors have completed the 
PRISMA reporting checklist. Available at https://dx.doi. org/10.21037/gs-21-618

Conflicts of Interest: All authors have completed the ICMJE uniform disclosure form (available at https://dx.doi. org/10.21037/gs-21-618). The authors have no conflicts of interest to declare.

Ethical Statement: The authors are accountable for all aspects of the work in ensuring that questions related to the accuracy or integrity of any part of the work are appropriately investigated and resolved.

Open Access Statement: This is an Open Access article distributed in accordance with the Creative Commons Attribution-NonCommercial-NoDerivs 4.0 International License (CC BY-NC-ND 4.0), which permits the noncommercial replication and distribution of the article with the strict proviso that no changes or edits are made and the original work is properly cited (including links to both the formal publication through the relevant DOI and the license). See: https://creativecommons.org/licenses/by-nc-nd/4.0/.

\section{References}

1. Guihard B, Chollet-Xémard C, Lakhnati P, et al. Effect of rocuronium vs succinylcholine on endotracheal intubation success rate among patients undergoing out-of-hospital rapid sequence intubation: a randomized clinical trial. JAMA 2019;322:2303-12.

2. Gualniera P, Scurria S, Mondello C, et al. Narrative review of proving the causal link of recurrent laryngeal nerve injury and thyroidectomy: a medico legal appraisal. Gland Surg 2020;9:1564-72.

3. Fábián ÁI, Csernoch V, Tassonyi E, et al. The effect of magnesium on the reversal of rocuronium-induced neuromuscular block with sugammadex: an ex vivo laboratory study. BMC Anesthesiol 2019;19:64.

4. FitzGerald H, Anderson E, Anderson LR, et al. Effectiveness of deep general anesthesia compared to the standard depth of general anesthesia for acute postoperative pain and patient safety: a systematic review protocol. JBI Database System Rev Implement Rep 2019;17:2129-35.

5. Liu X, Ji J, Zhao GQ. General anesthesia affecting on developing brain: evidence from animal to clinical research. J Anesth 2020;34:765-72.

6. Van Gasse AL, Elst J, Bridts CH, et al. Rocuronium hypersensitivity: does off-target occupation of the MRGPRX2 receptor play a role? J Allergy Clin Immunol Pract 2019;7:998-1003.

7. Choi SN, Jang YE, Lee JH, et al. Comparison of rocuronium requirement in children with continuous infusion versus intermittent bolus: a randomised controlled trial. Eur J Anaesthesiol 2019;36:194-9.

8. Dhanarisi J, Shihana F, Harju K, et al. A pilot clinical study of the neuromuscular blocker rocuronium to reduce the duration of ventilation after organophosphorus insecticide poisoning. Clin Toxicol (Phila) 2020;58:254-61.

9. Lu IC, Tsai CJ, Wu CW, et al. A comparative study between 1 and 2 effective doses of rocuronium for intraoperative neuromonitoring during thyroid surgery. Surgery 2011;149:543-8.

10. Hurford WE, Eckman MH, Welge JA. Data and metaanalysis for choosing sugammadex or neostigmine for routine reversal of rocuronium block in adult patients. Data Brief 2020;32:106241.

11. April MD, Arana A, Pallin DJ, et al. Emergency department intubation success with succinylcholine versus rocuronium: a national emergency airway registry study. Ann Emerg Med 2018;72:645-53.

12. Banasiewicz T, Meissner W, Pyda P, et al. Local anesthesia in thyroid surgery--own experience and literature review. Pol Przegl Chir 2011;83:264-70.

13. Barsotti G, Asti M, Giani E, et al. Effect of topical ophthalmic instillation of rocuronium bromide on the intraocular pressure of kestrels (Falco tinnunculus) and little owls (Athene noctuae). J Am Vet Med Assoc 2019;255:1359-64.

14. Dong J, Gao L, Lu W, et al. Pharmacological interventions for acceleration of the onset time of rocuronium: a metaanalysis. PLoS One 2014;9:e114231.

15. Goeller JK, Bhalla T, Tobias JD. Combined use of neuraxial and general anesthesia during major abdominal procedures in neonates and infants. Paediatr Anaesth 2014;24:553-60.

16. Han YD, Liang F, Chen P. Dosage effect of rocuronium on intraoperative neuromonitoring in patients undergoing thyroid surgery. Cell Biochem Biophys 2015;71:143-6.

17. Hawkins RJ, Swanson B, Kremer MJ. An integrative review of factors related to patient satisfaction with general anesthesia care. AORN J 2012;96:368-76.

18. Kanazawa M, Sato Boku A, Okumura Y, et al. The effect of various dilute administration of rocuronium bromide on both vascular pain and pharmacologic onset: a randomized controlled trial. BMC Anesthesiol 2019;19:76. 
19. Palanisamy A, Giri T. Reduced severe acute respiratory syndrome coronavirus 2 entry factors and enhanced innate immune gene expression in the nasal epithelium of pregnant rats. Am J Obstet Gynecol 2021;224:118-20.

20. Prabhakar H, Singh GP, Ali Z, et al. Pharmacological and non-pharmacological interventions for reducing rocuronium bromide induced pain on injection in children and adults. Cochrane Database Syst Rev 2016;2:CD009346.

21. Tachikawa M, Asai T, Okuda Y. Rocuronium Bromide Intravenous Solution Maruishi ${ }^{\circledR}$ is more suitable than ESLAX Intravenous ${ }^{\circledR}$ during rapid-sequence induction of anesthesia. J Anesth 2019;33:600-3.

22. Hashimoto M, Sato Boku A, Tachi N, et al. Two cases of rocuronium-induced anaphylaxis/anaphylactic shock successfully treated with sugammadex. Anesth Prog 2019;66:151-5.

23. Kannan S, Surhonne NS, Kumar RC, et al. Effects of bilateral superficial cervical plexus block on sevoflurane consumption during thyroid surgery under entropyguided general anesthesia: a prospective randomized study. Korean J Anesthesiol 2018;71:141-8.

24. Matsui M, Konishi J, Suzuki T, et al. Reversibility of

Cite this article as: Zhong B, Gao G, Sun D, Zhang L. A systematic review and meta-analysis of the effect of different doses of rocuronium for general anesthesia on thyroid surgery. Gland Surg 2021;10(12):3241-3251. doi: 10.21037/gs-21-618 rocuronium-induced deep neuromuscular block with sugammadex in infants and children-A randomized study. Biol Pharm Bull 2019;42:1637-40.

25. Petritz OA, Westermeyer HD, Whitehead MC, et al. Effective mydriasis in juvenile loggerhead turtles (Caretta caretta) following topical administration of rocuronium bromide and $10 \%$ phenylephrine. Vet Ophthalmol 2020;23:37-43.

26. Takagi S, Sugaya N, Kiuchi N, et al. High-dose rocuronium-induced paralysis of the adductor pollicis muscle facilitates detection of the timing for tracheal intubation in elderly patients: a randomized double-blind study. J Anesth 2020;34:876-80.

27. Garofalo E, Bruni A, Scalzi G, et al. Low-Dose of Rocuronium During Thyroid Surgery: Effects on Intraoperative Nerve-Monitoring and Intubation. J Surg Res 2021;265:131-8.

28. Aragón-Benedí C, Visiedo-Sánchez S, Pascual-Bellosta A, et al. Study of Rocuronium-Sugammadex as an Alternative to Succinylcholine-Cisatracurium in Microlaryngeal Surgery. Laryngoscope 2021;131:E212-8.

(English Language Editor: B. Draper) 